\title{
Perceptions and Use of Native Forests in the Arid Chaco of Córdoba, Argentina
}

\author{
Cecilia Trillo, Sonia Colantonio, and Leonardo Galetto
}

\section{Research}

\begin{abstract}
The human population in the arid Chaco forests of Argentina is composed mainly of stockmen carrying out ancestral practices. Plant uses in this biogeographic region are relatively well known, but the forest perception by local populations was not studied so far. A total of 77 stockmen and other local salaried people were interviewed with semi-structured interviews, and herbarium specimens were produced with the informants. A Likert scale was used to obtain perceptions of the forest value. We registered 124 plant species, particularly forage woody plants, which have a variety of uses. More than 100 species overlapped with a survey of the region more than one century ago. Stockmen carry out ancestral practices such as cutting, collecting, and storing dry fruit, creating elaborate cercos, and know substantially more forage plants. The results suggest that the local population as a whole has a positive perception of the forests, regardless of their main occupation, and needs the forest to reinforce stockmen's traditions (criollos) through daily activities.
\end{abstract}

\section{Introduction}

Traditional ecological knowledge can be defined as the cumulative body of knowledge, practices, and beliefs evolved by adaptive processes and handed down through generations by cultural transmission of the relationships among living organisms (including humans) with their environment (Berkes 1999). This concept implies environmental perceptions shaped by religion, ethics, and other sets of beliefs. In this context, local forest inhabitants use their traditional ecological knowledge for obtaining multiple services and resources that support their daily activities. In addition, forests provide both urban and rural populations with products from different species, such as building and crafts materials, fuel, dietary supplements, and medicinal plants (Cunningham 2001). The most common products from South American forests are firewood, coal, and quality woods for different purposes. However, there are many non-timber goods used for local subsistence or for national and international trading (FAO 2009, Phillips et al. 1994).

Subtropical Chaco forests have been dramatically reduced, mainly by soybean expansion (Zak et al. 2008). The residents of the dry Chaco woodlands have different occupations (e.g., stockmen and urban or semi-urban people, with salaried employees or small-scale traders). For their subsistence, they usually sustain extensive cattle farming and non-irrigated crops applying traditional knowledge and techniques (Trillo et al. 2010). Forest resources seem to be essential for stockmen mainly because of the agricultural expansion into the driest and most marginal areas of this region. Previous ethnobotanical research focused on plant uses such as edibility (Arenas 1999), healing (Arenas 2000, Barboza et al. 2009, Filipov 1997, Idoyaga Molina 2001, Trillo et al. 2010), dye (Trillo et al.

\section{Correspondence \\ Cecilia Trillo, Cát. Diversidad Vegetal II, FCEFyN, Universi- dad Nacional de Córdoba, ARGENTINA. ceciliatrillo1@gmail.com \\ Sonia Colantonio, Cát. Antropología Biológica y Cultural, FCEFyN, Universidad Nacional de Córdoba, CONICET, ARGENTINA. \\ Leonardo Galetto, Cát. Diversidad Vegetal II, FCEFyN, Uni- versidad Nacional de Córdoba e IMBIV (UNC-CONI- CET), ARGENTINA.}

Ethnobotany Research \& Applications 12:497-510 (2014)

Published: 21 November 2014 
2007), or forage (Muiño 2010, Scarpa 2007). Few studies considered the economic value of Chaco plants (Muiño 2010, Scarpa 2007).

Although local residents are linked in different ways to the remaining forests and may have different views regarding forest goods, perceptions of the forest have not yet been studied. According to Padua (1994), it is assumed that perceptions represent a nexus between the individual psychological condition (i.e., motivational, emotional, perceptual, and cognitive processes) and the external objects (forest plants, in this case). For this biogeographic region the link between the relatively well known plant uses and the forest perception by resident people was not previously studied.

There are few historic documents that mention the knowledge and/or techniques used for rural activities in the Chaco region of Córdoba province. The traditional economic activity in the dry Chaco woodlands was the extensive farming of cattle, goats, and sheep (Celton 1993, Río \& Achával 1905), with basic practices associated with the management of forage plants and cattle (Díaz 2007). The present ethnobotanical study about the local knowledge of the forest and particularly about the forage plants or the associated practices to obtain resources in this marginal landscape, aims to contribute to fill this documentation gap.

The main objective of this study was to compare plant uses and forest perceptions between the local populations with different occupations (stockmen and other occupations) that determine a different daily relationship with the forests. The inhabitants' perception of the forest would be a key point to better understand their daily practices, forest management, and conservation approaches. This study intended to (1) record the plant uses of residents of the Chaco forests in its western region and to compare the persistence of this knowledge with data from one century ago, (2) compare the forest perceptions between stockmen and other occupations, and (3) describe the traditional practices of stockmen associated with forage plants.

\section{Materials and Methods}

\section{Study area}

The Guasapampa valley (Minas department, Córdoba province) is a rural area located at $31^{\circ} 0^{\prime} \mathrm{S}$ and $65^{\circ} 22^{\prime} \mathrm{W}$. It has an extent of $20 \mathrm{~km}$ long and $6 \mathrm{~km}$ wide and an altitude ranging from 540 to 750 masl. The annual average temperature is $18^{\circ} \mathrm{C}$, with an annual precipitation of $400-500$ $\mathrm{mm}$, occurring mainly during summer (Di Tada \& Bucher 1996). There are three main villages: La Playa, Guasapampa, and Totora Huasi, with 163, 193, and 24 inhabitants, respectively (Figure 1).
The Minas department is one of the more economically relegated regions within the province of Córdoba, with critical levels of illiteracy and unemployment (González 1999). The main productive systems are extensive cattle farming, forest exploitation, mining, and non-irrigated agriculture (Bergamín 1992, Trillo et al. 2010).

The plant communities are representative of the Chaco Seco (i.e., dry) phytogeographic region (Cabrera 1976). The flora consists of woody species such as Aspidosperma quebracho-blanco Schltdl., Schinopsis lorentzii (Griseb.) Engl., Lithrea molleoides (Vell.) Engl., Prosopis flexuosa DC., Acacia aroma Gillies ex Hook. et Arn., Flourensia oolepis S.F.Blake, Ruprechtia apetala Wedd., Celtis iguanaea (Jacq.) Sarg., Senegalia gilliesii (Steud.) Seigler \& Ebinger, and Larrea divaricata Cav. Most of this region has been disturbed mainly by logging, cattle farming, and fires during the last 30 years. Secondary forests are characterized by few trees and many shrubs (Cabido \& Pacha 2002), including A. aroma, Acacia caven (Molina) Molina, Condalia microphylla Cav., F. oolepis, Croton lachnostachyus Baill., Aloysia gratissima (Gillies \& Hook.) Tronc., Ephedra triandra Tul., and Heterothalamus alienus (Spreng.) Kuntze., and have showed major recent changes in their structure (Zak \& Cabido 2002).

\section{The population}

Inhabitants of the Guasapampa Valley identify themselves as criollos. Criollos are the descendants of Europeans and regional ethnic groups, catholic and Spanish speakers (Celton 1993). The population of the northwestern Córdoba province in the pre-Hispanic $16^{\text {th }}$ century was constituted of 600 pueblos or regional ethnic groups according to the Spanish conqueror at $16^{\text {th }}$ century, Jerónimo Luis de Cabrera (Celton 1993). The families lived in little towns near rivers and developed basic agriculture (e.g., corn, pumpkins), weaving, and ceramics. When the Spanish began to settle in the area, native ethnic groups began a process of dispersion and erosion of their cultural and administrative organization and eventually became mixed people (Celton 1993). Today, their descendants carry out activities related to livestock farming, subsistence agriculture, and textile production. They live in houses called ranchos, which are surrounded by little orchard-gardens and rustic fences called cercos to protect their sheep and goats.

The historic continuity of the practices associated with stock raising was documented by Río and Achával (1905). These geographers reviewed the economic activities in the province since the $17^{\text {th }}$ century and included in their research the study of J. Hieronymus' Plantae Diaphorae Florae Argentinae, published in the Bulletin of National Science Academy in 1882, which described the nutritional, medical, and other uses of native plants. 


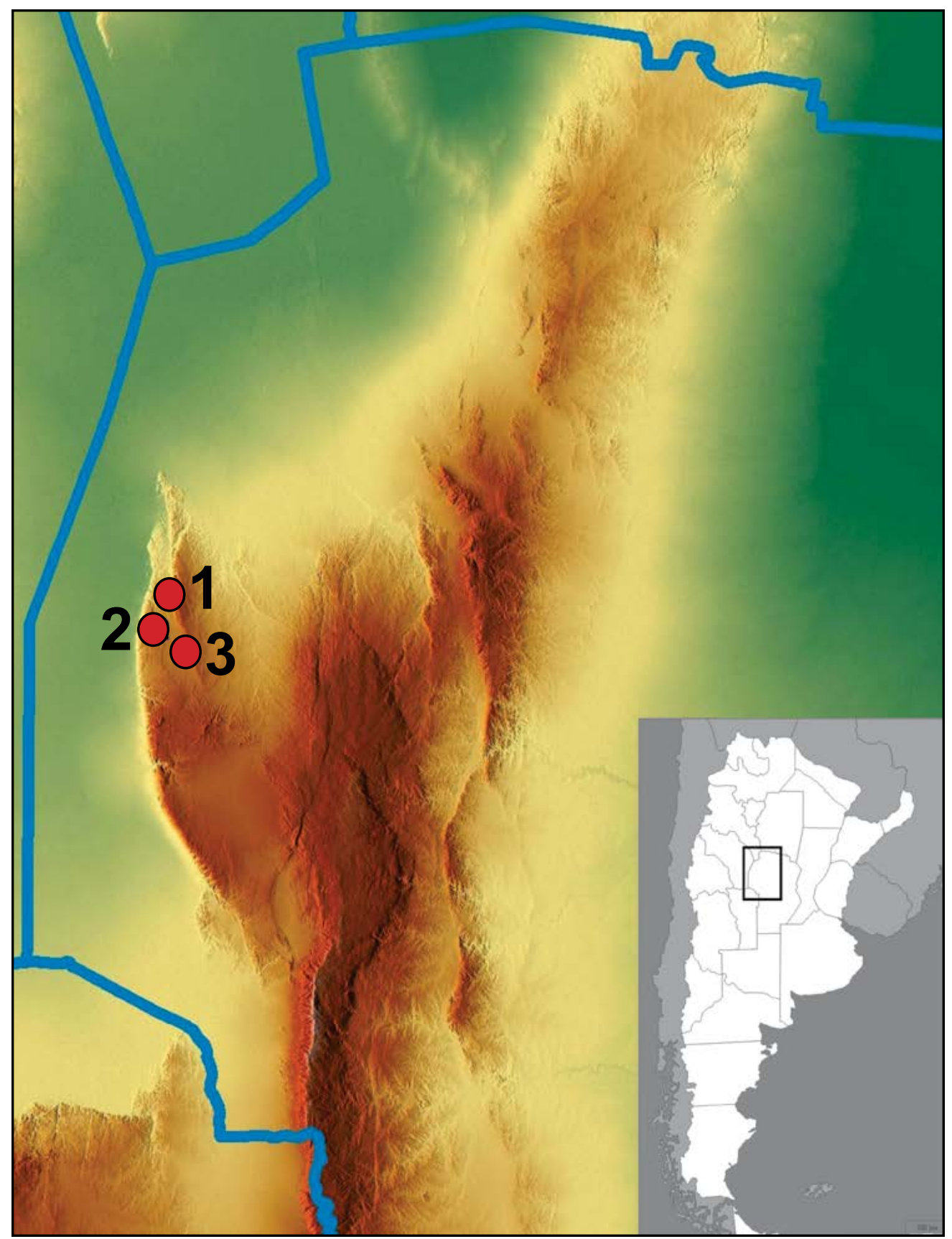

Figure 1. Study area and main mountain ranges (sierras) and villages (1: Totora Huasi, 2: La Playa, and 3: Guasapampa) in the Guasapampa valley, Minas department, Córdoba province, Argentina.

In recent times economic progress has generated new opportunities so many inhabitants of this rural area now have different occupations. Interviewees were classified as "stockmen" or with a "different occupation" based on both their occupation and their main monetary income. A "stockman" was defined as a man or woman who owns cattle, goats, horses, or sheep, and sometimes sells medicinal plants, cheese, wool, wood, or honey. Every day, they walk at least a few kilometers into the forest to feed their animals. A "different occupation" was defined as a man or woman who works in the villages' urban zone (e.g., at a school, a police station, a store, or a mine). These categories were usually selfdefined by the interviewees, but in those few cases that they did not do so, we categorized them based on their daily activities and their most important income.

\section{Data collection}

Most of the adult residents in each village were interviewed (80\%). Data were collected through semistructured interviews (Bernard 1995, Padua 1994) about daily activities, plant uses, forest perceptions, and their traditions. A total of 77 inhabitants were interviewed, both men and women, from 16 to 70 years old: 33 stockmen (12 men and 21 women) and 44 having different occupations (16 men and 28 women). Each informant gave data about gender, age, family, place of birth, parents' origin, principal activities for their subsistence and income, daily route for those activities, water access for human and stock consumption, forest access, plant collection, etc.

Perceptions of the forest and the natural environment were quantified using ordinal data with a Likert-type scale (Likert 1932) which was used to obtain perceptions of the forest as being the natural environment offered 
to residents. In Likert-type scale questions, the informant gave an opinion or stance about one item (i.e., different essential resources that they need for subsistence: forage plants for animals, medicine, furniture, etc.). Six questions were specifically asked to quantify their forest perception (i.e., importance of the forest to provide forage, medical, dye, and veterinary plants, and also other products or pleasure sensations). The term "forage" was utilized to refer to all the food used to feed animals (i.e., products of vegetable origin and used without transformation, except for dehydration and milling). These questions were numerically quantified by means of three options in descending grade of importance: very important, important, and less important (3,2, and 1, respectively). Finally, the informant examined the herbarium specimens to check and/or confirm plant uses. The herbarium collections consisted of 99 plant species that were created with the help of key informants from the three towns. These exsiccatae are deposited at the Botany Museum of the National University of Córdoba (CORD).

Using the information documented in the database from Río and Achával (1905), we have compared the plants cited by the current inhabitants with those plants used over a century ago. This methodological approach is proposed by Medeiros (2009).

\section{Statistical analyses}

SPSS 11.5 statistical package was used to compared the number of plants with different uses known by stockmen and inhabitants with different occupations using t-tests. Wilcoxon tests were run to compare the forest perceptions of stockmen and salaried employees. The modes obtained for each question/category were used to compare data (Padua 1994).

The cultural significance that inhabitants attributed to different plant resources was analysed using a community valuation. This analysis was intended to estimate the cultural importance of the different plants and to determine the agreement among the local people regarding the usefulness of such plants. The cultural value was calculated for each species of the herbarium following the formula proposed by Phillips and Gentry (1993): $V U_{\text {is }}=\sum\left(U_{\text {is }} / n_{\text {is }}\right)$. The use value $\left(\mathrm{VU}_{\mathrm{is}}\right)$ was calculated by totalling all the uses mentioned for a given plant in each event by the interviewees $\left(\mathrm{U}_{\mathrm{is}}\right)$, and then dividing this value by the total number of events in which the informants gave information on the species $\left(n_{i s}\right)$. This index is interpreted as a measure of the importance of these plants for the residents of this region. It also shows the level of agreement among local people about the utility of these plants.

\section{Results}

\section{Plant uses}

A total of 123 plant species from 47 botanical families were mentioned by informants in relation to the different uses (Table 1). One species each of lichen, fungus, insect, and reptile were also mentioned. The average number of cited species is 37.5 (range: $8-85$ ). Most residents mentioned 3-44 species; few of them knew more than 44 or less than 3 species.

A small number of species $(n=20)$ presented a wide consensus (i.e., 50 to $90 \%$ of the inhabitants), but most spe-

Table 1. Useful plants and other organisms mentioned by residents in Guasapampa Valley, Córdoba, Argentina. Uses: F-forage (106 spp.), M-medical (83 spp.), V-veterinarian (34 spp.), N-nutritional (17 spp.), D-dyes (42 spp.), Fl-fuel (19 spp.), T-tools (7 spp.), Fu-furniture (4 spp.), C-corrals (10 spp.), Sh-shade (6 spp.), Ma-magical (2 spp.), Mo-mordant (2 spp.), S-silos (1 sp.), So-soap (1 sp), Cl-clean the water (1 sp). The 20 highest cultural use values are in bold. Green font indicates that a species was previously mentioned in Rio and Achával (1905) with an asterisk $\left(^{*}\right)$ indicating the use category from 1905.

\begin{tabular}{|c|c|c|c|c|}
\hline Scientific name & Vernacular name & Consent & $\begin{array}{l}\text { Use } \\
\text { value }\end{array}$ & Use(s) \\
\hline \multicolumn{5}{|l|}{ Parmeliaceae (lichen) } \\
\hline Usnea amblyoclada (Müll.Arg.) Zahlbr & barba de piedra & 2 & - & $M^{*}, D$ \\
\hline \multicolumn{5}{|l|}{ Anemiaceae } \\
\hline Anemia tomentosa (Savigny) Sw. & doradilla & 25 & 1.05 & $\mathrm{~F}, \mathrm{M}^{*}$ \\
\hline \multicolumn{5}{|l|}{ Equisetaceae } \\
\hline Equisetum giganteum L. & cola de caballo & 5 & - & $\mathrm{M}^{*}$ \\
\hline \multicolumn{5}{|l|}{ Lycopodiaceae } \\
\hline Phlegmariurus saururus (Lam.) B.Øllg. & $\begin{array}{l}\text { cola de } \\
\text { quirquincho }\end{array}$ & 1 & - & $M^{*}$ \\
\hline \multicolumn{5}{|l|}{ Pteridaceae } \\
\hline Argyrochosma nivea (Poir.) Winham & culandrillo & 5 & 0.1 & $\mathrm{~F}, \mathrm{M}^{*}$ \\
\hline
\end{tabular}


Trillo et al. - Perceptions and Use of Native Forests in the Arid Chaco of Córdoba, Argentina

\begin{tabular}{|c|c|c|c|c|}
\hline \multicolumn{5}{|l|}{ Ephedraceae } \\
\hline Ephedra triandra Tul. & tramontana & 24 & 0.66 & $\mathrm{~F}, \mathrm{M}^{*}$ \\
\hline \multicolumn{5}{|l|}{ Amaranthaceae } \\
\hline Alternanthera pungens Kunth & yerba del pollo & 20 & 0.64 & $F, M^{*}$ \\
\hline Amaranthus hybridus L. & $\begin{array}{l}\text { amaranto, yuyo } \\
\text { colorado }\end{array}$ & 8 & 0.11 & $\mathrm{~F}$ \\
\hline Atriplex undulata (Moq.) D.Dietr. & cachiyuyo & 38 & 0.75 & $\begin{array}{l}\mathrm{F}, \mathrm{M}, \\
D^{*}, M A\end{array}$ \\
\hline Chenopodium album L. & yerba de la perdiz & 2 & 0.16 & $\mathrm{~F}, \mathrm{M}^{*}$ \\
\hline Dysphania ambrosioides (L.) Mosyakin \& Clemants & paico & 17 & 0.51 & $\mathrm{~F}, \mathrm{M}^{*}$ \\
\hline \multicolumn{5}{|l|}{ Anacardiaceae } \\
\hline Lithraea molleoides (Vell.) Engl. & molle & 33 & 0.95 & $\begin{array}{l}\mathrm{F}, \mathrm{M}^{*}, \mathrm{D} \\
\mathrm{V}, \mathrm{N}^{*}\end{array}$ \\
\hline Schinopsis lorentzii (Griseb.) Engl. & orco quebracho & 32 & 0.91 & $\begin{array}{l}\mathrm{F}, \mathrm{M}^{*}, \mathrm{D} \\
\mathrm{Fu}^{*}, \mathrm{FI} \\
\end{array}$ \\
\hline Schinus areira L. & aguaribay & 1 & - & $\mathrm{M}^{*}$ \\
\hline Schinus fasciculata (Griseb.) I.M.Johnst. & $\begin{array}{l}\text { molle pispo } \\
\text { o pispito }\end{array}$ & 37 & 0.55 & $\begin{array}{l}\text { F, D, Sh, } \\
\text { Fl, C }\end{array}$ \\
\hline \multicolumn{5}{|l|}{ Apocynaceae } \\
\hline Araujia brachystephana (Griseb.) Fontella \& Goyder & tasi & 1 & 0.57 & $\mathrm{~F}, \mathrm{M}^{*}, \mathrm{~N}^{*}$ \\
\hline Aspidosperma quebracho-blanco Schltdl. & quebracho blanco & 9 & 0.65 & $\begin{array}{l}\mathrm{F}, \mathrm{M}^{*}, \mathrm{D}^{*} \\
\mathrm{~V}, \mathrm{Mo}, \mathrm{C} \\
\mathrm{Fl}^{*}\end{array}$ \\
\hline Vallesia glabra (Cav.) Link. & ancoche, coquillo & 15 & 0.41 & $\begin{array}{l}\mathrm{F}, \mathrm{N}, \mathrm{M}^{*} \\
\mathrm{~V}, \mathrm{~T}\end{array}$ \\
\hline \multicolumn{5}{|l|}{ Aristolochiaceae } \\
\hline Aristolochia argentina Griseb. & charrúa & 7 & 0.27 & $\mathrm{~F}, \mathrm{M}^{*}$ \\
\hline \multicolumn{5}{|l|}{ Asteraceae } \\
\hline Achyrocline sp. & $\begin{array}{l}\text { lavanda del } \\
\text { campo }\end{array}$ & 2 & 0.14 & $F, M$ \\
\hline Achyrocline satureioides (Lam.) DC. & vira-vira & 38 & 0.95 & $\mathrm{~F}, \mathrm{M}^{*}, \mathrm{D}$ \\
\hline Ambrosia tenuifolia Spreng. & altamisa & 9 & 0.29 & $\mathrm{~F}, \mathrm{M}^{*}$ \\
\hline Artemisia douglasiana Besser ex Besser & matico & 3 & 0.19 & $\mathrm{~F}, \mathrm{M}^{*}$ \\
\hline Baccharis articulata (Lam.) Pers. & carqueja & 11 & 0.76 & $\mathrm{~F}, \mathrm{M}^{*}, \mathrm{D}$ \\
\hline Baccharis salicina Torr. \& A.Gray & chilca amarga & 11 & 0.43 & $\mathrm{~F}, \mathrm{M}^{*}, \mathrm{D}, \mathrm{T}$ \\
\hline Cyclolepis genistoides D.Don & palo azul & 29 & 1.16 & $\mathrm{~F}, \mathrm{M}^{*}, \mathrm{D}$ \\
\hline Flaveria bidentis (L.) Kuntze & fique o balda & 13 & 0.49 & $\mathrm{~F}, \mathrm{M}^{*}, \mathrm{D}^{*}$ \\
\hline Flourensia oolepis S.F.Blake & chilca del campo & 18 & 0.23 & $\mathrm{~F}, \mathrm{D}^{*}$ \\
\hline Gaillardia megapotamica (Spreng.) Baker & topasaire & 15 & 0.67 & $\mathrm{~F}, \mathrm{M}^{*}$ \\
\hline Grindelia pulchella Dunal & tiñe rosado & 1 & 0.07 & $\mathrm{~F}, \mathrm{D}^{*}$ \\
\hline Heterothalamus alienus (Spreng.) Kuntze & romerillo & 1 & - & $D^{*}$ \\
\hline Jungia polita Griseb. & zarzaparrilla & 33 & 1.07 & $\mathrm{~F}, \mathrm{M}^{*}$ \\
\hline Pluchea dodonaeifolia (Hook. \& Arn.) H.Rob. \& Cuatrec. & $\begin{array}{l}\text { suncho, chilca } \\
\text { dulce }\end{array}$ & 11 & 0.43 & $\begin{array}{l}\mathrm{F}, \mathrm{M}^{*}, \mathrm{D} \\
\mathrm{Mo}, \mathrm{T}\end{array}$ \\
\hline Pluchea sagittalis (Lam.) Cabrera & lucera & 1 & 0.03 & $\mathrm{~F}, \mathrm{M}^{*}$ \\
\hline Porophyllum obscurum (Spreng.) DC. & yerba del venado & 1 & 0.04 & $\mathrm{~F}, \mathrm{M}^{*}$ \\
\hline
\end{tabular}




\begin{tabular}{|c|c|c|c|c|}
\hline Schkuhria pinnata (Lam.) Kuntze ex Thell. & matapulga & 17 & 0.77 & $\begin{array}{l}\mathrm{F}, \mathrm{M}^{*}, \mathrm{~V}^{*}, \\
\mathrm{~T}\end{array}$ \\
\hline Senecio pampeanus Cabrera & pichanilla & 4 & 0.1 & $\mathrm{~F}, \mathrm{~V}$ \\
\hline Taraxacum officinale F.H.Wigg. & diente de león & 1 & - & $\mathrm{M}^{*}$ \\
\hline Thymophylla pentachaeta (DC.) Small & guillermito & 32 & 0.91 & $\mathrm{~F}, \mathrm{M}^{*}$ \\
\hline Trixis divaricata (Kunth) Spreng. & contrahierba & 29 & 1.07 & $\begin{array}{l}\mathrm{F}, \mathrm{M}^{*}, \mathrm{D} \\
\mathrm{V}, \mathrm{Ma}\end{array}$ \\
\hline Verbesina encelioides (Cav.) Benth. \& Hook.f. ex A.Gray & mirasol & 11 & 0.19 & $\mathrm{~F}, \mathrm{M}^{*}$ \\
\hline Xanthium cavanillesii Schouw ex Didr. & abrojo & 4 & 0.08 & $\mathrm{~F}, \mathrm{~V}$ \\
\hline Xanthium spinosum L. & cepacaballos & 6 & 0.35 & $\mathrm{~F}, \mathrm{M}^{*}, \mathrm{~V}$ \\
\hline Zinnia peruviana (L.) L. & clavelillo & 8 & 0.18 & F, D \\
\hline \multicolumn{5}{|l|}{ Bignoniaceae } \\
\hline Amphilophium carolinae (Lindl.) L.G.Lohmann & cancana & 9 & 0.19 & $\mathrm{~F}$ \\
\hline \multicolumn{5}{|l|}{ Boraginaceae } \\
\hline Heliotropium amplexicaule Vahl & yerba meona & 12 & 0.26 & $\mathrm{~F}, \mathrm{M}^{*}$ \\
\hline Nama undulata Kunth & matagusano & 7 & 0.48 & $F, M^{*}, V^{*}$ \\
\hline \multicolumn{5}{|l|}{ Bromeliaceae } \\
\hline Dyckia floribunda Griseb. & $\begin{array}{l}\text { penca de las } \\
\text { piedras }\end{array}$ & 9 & 0.16 & $\mathrm{~F}$ \\
\hline Tillandsia aizoides Mez & suelda & - & - & $\mathrm{F}$ \\
\hline Tillandsia capillaris Ruiz \& Pav. & suelda & 46 & 0.79 & $\mathrm{~F}$ \\
\hline Tillandsia duratii Vis. & suelda & - & - & $F, M^{*}, N$ \\
\hline \multicolumn{5}{|l|}{ Buddlejaceae } \\
\hline Buddleja cordobensis Griseb. & $\begin{array}{l}\text { pulmonaria, } \\
\text { sanalotodo }\end{array}$ & 10 & 0.2 & $\mathrm{~F}, \mathrm{M}^{*}$ \\
\hline \multicolumn{5}{|l|}{ Cactaceae } \\
\hline Acanthocalycium spiniflorum (K.Schum.) Backeb. & penca & - & - & $\mathrm{F}$ \\
\hline Gymnocalycium ochoterenae Backeb. & penca & 39 & 0.58 & $\mathrm{~F}$ \\
\hline Opuntia ficus-indica (L.) Mill. & tuna & 30 & - & $\mathrm{F}^{*}, \mathrm{M}^{*}, \mathrm{~N}$ \\
\hline Opuntia sulphurea Gillies ex Salm-Dyck & penca del burro & 30 & - & $\mathrm{F}$ \\
\hline \multicolumn{5}{|l|}{ Cannabaceae } \\
\hline Celtis iguanaea (Jacq.) Sarg. & tala & 33 & 0.88 & $\begin{array}{l}\mathrm{F}, \mathrm{M}^{*}, \mathrm{D}, \\
\mathrm{V}, \mathrm{Sh}, \mathrm{Fl}^{*}, \\
\mathrm{~N}^{*}, \mathrm{C}\end{array}$ \\
\hline \multicolumn{5}{|l|}{ Capparaceae } \\
\hline Atamisquea emarginata Miers ex Hook. \& Arn. & atamisqui & 31 & 0.71 & $\mathrm{~F}, \mathrm{M}^{*}$ \\
\hline \multicolumn{5}{|l|}{ Santalaceae } \\
\hline Jodina rhombifolia (Hook. \& Arn.) Reissek & peje & 6 & 0.43 & $\begin{array}{l}\mathrm{F}, \mathrm{M}^{*}, \mathrm{D} \\
\mathrm{V}, \mathrm{Sh}, \mathrm{Fl}^{*}\end{array}$ \\
\hline \multicolumn{5}{|l|}{ Commelinaceae } \\
\hline Commelina erecta L. & Santa Lucía & 8 & 0.16 & $\mathrm{~F}, \mathrm{M}^{*}$ \\
\hline \multicolumn{5}{|l|}{ Cucurbitaceae } \\
\hline Cayaponia citrullifolia (Griseb.) Cogn. ex Griseb. & $\begin{array}{l}\text { sandía de } \\
\text { la víbora }\end{array}$ & 8 & 0.16 & $\mathrm{~F}, \mathrm{M}, \mathrm{V}$ \\
\hline
\end{tabular}




\begin{tabular}{|c|c|c|c|c|}
\hline \multicolumn{5}{|l|}{ Euphorbiaceae } \\
\hline Croton lachnostachyus Baill. & bálsamo & 9 & 0.22 & $\mathrm{~F}, \mathrm{M}^{*}$ \\
\hline \multicolumn{5}{|l|}{ Fabaceae } \\
\hline Acacia aroma Gillies ex Hook. \& Arn. & tusca & 49 & 1.27 & $\begin{array}{l}\mathrm{F}, \mathrm{M}^{*}, \mathrm{D} \\
\mathrm{V}, \mathrm{Fl}^{*}, \mathrm{C}\end{array}$ \\
\hline Acacia caven (Molina) Molina & espinillo & 34 & 0.67 & $\begin{array}{l}\mathrm{F}, \mathrm{M}^{*}, \mathrm{D} \\
\mathrm{V}, \mathrm{FI}\end{array}$ \\
\hline Acacia praecox Griseb. & garabato hembra & 8 & 0.23 & $\mathrm{~F}, \mathrm{M}, \mathrm{D}, \mathrm{FI}$ \\
\hline Caesalpinia gilliesii (Hook.) D.Dietr. & $\begin{array}{l}\text { lagaña de } \\
\text { perro, chosni }\end{array}$ & 5 & 0.12 & $\begin{array}{l}\mathrm{F}, \mathrm{M}, \mathrm{D}^{*} \\
\mathrm{~V}\end{array}$ \\
\hline Geoffroea decorticans (Hook. \& Arn.) Burkart & chañar & 35 & 1.48 & $\begin{array}{l}\mathrm{F}, \mathrm{M}^{*}, \mathrm{Fl}^{*}, \\
\mathrm{~N}^{*}\end{array}$ \\
\hline Medicago sativa L. & *alfalfa & 26 & - & $\mathrm{F}^{*}$ \\
\hline Parkinsonia praecox (Ruiz \& Pav.) Hawkins & brea & 1 & - & $\mathrm{V}$ \\
\hline Prosopis chilensis (Molina) Stuntz & árbol blanco & 49 & 1.51 & $\mathrm{FI}^{*}, \mathrm{~N}, \mathrm{C}$ \\
\hline Prosopis flexuosa DC. & árbol negro & 44 & 1.14 & $\begin{array}{l}\mathrm{F}, \mathrm{D}, \mathrm{V} \\
\mathrm{Fu}^{*}, \mathrm{Sh}\end{array}$ \\
\hline Prosopis torquata (Lag.) DC. & tintitaco & 25 & 0.49 & $\begin{array}{l}\mathrm{F}, \mathrm{M}^{*}, \mathrm{D}, \\
\mathrm{V}, \mathrm{Fu}^{*}, \\
\mathrm{Sh}, \mathrm{FI}^{*}, \mathrm{~N}, \\
\mathrm{C}\end{array}$ \\
\hline Senegalia gilliesii (Steud.) Seigler \& Ebinger & garabato macho & 17 & 0.28 & $\mathrm{~F}, \mathrm{FI}^{*}$ \\
\hline Senna aphylla (Cav.) H.S.Irwin \& Barneby & pichana & 1 & - & $\begin{array}{l}\mathrm{F}, \mathrm{M}^{*}, \mathrm{D}, \\
\mathrm{FI}, \mathrm{C}\end{array}$ \\
\hline Senna corymbosa (Lam.) H.S.Irwin \& Barneby & falso cafeto, sen & 3 & 0.05 & $\mathrm{~F}, \mathrm{M}^{*}$ \\
\hline \multicolumn{5}{|l|}{ Gentianaceae } \\
\hline Gentianella sp. & nencia & 1 & - & $\mathrm{M}^{*}$ \\
\hline \multicolumn{5}{|l|}{ Hydnoraceae } \\
\hline Prosopanche americana (R.Br.) Baill. & guaycurú & 2 & & $\mathrm{M}^{*}$ \\
\hline \multicolumn{5}{|l|}{ Lamiaceae } \\
\hline Hedeoma multiflora Benth. & tomillo & 14 & - & $\mathrm{M}^{*}$ \\
\hline Marrubium vulgare L. & yerba del sapo & 19 & 0.94 & $F, M^{*}$ \\
\hline Minthostachys verticillata (Griseb.) Epling & peperina & 8 & - & $\mathrm{M}^{*}$ \\
\hline \multicolumn{5}{|l|}{ Loranthaceae } \\
\hline Ligaria cuneifolia (Ruiz \& Pav.) Tiegh. & liga & 45 & 0.93 & $\begin{array}{l}\mathrm{F}, \mathrm{M}^{*} \\
\mathrm{D}, \mathrm{V}\end{array}$ \\
\hline \multicolumn{5}{|l|}{ Lythraceae } \\
\hline Heimia salicifolia (Kunth) Link & quiebra arado & 27 & 0.39 & $\mathrm{~F}, \mathrm{M}^{*}, \mathrm{Cl}$ \\
\hline \multicolumn{5}{|l|}{ Malvaceae } \\
\hline Sida glabra Mill. & yerba del potro & 1 & 0.19 & $\mathrm{~F}$ \\
\hline Sphaeralcea cordobensis Krapov. & malva & 14 & 0.52 & $\mathrm{~F}, \mathrm{M}^{*}, \mathrm{Cl}$ \\
\hline \multicolumn{5}{|l|}{ Nyctaginaceae } \\
\hline Bougainvillea stipitata Griseb. & tala falso & 10 & 0.3 & $\begin{array}{l}\mathrm{F}, \mathrm{Fl}^{*} \\
\mathrm{~N}, \mathrm{~T}^{*}\end{array}$ \\
\hline
\end{tabular}




\begin{tabular}{|c|c|c|c|c|}
\hline \multicolumn{5}{|l|}{ Olacaceae } \\
\hline Ximena americana L. & albarillo & 29 & 0.55 & $F, D^{*}, N^{*}$ \\
\hline \multicolumn{5}{|l|}{ Papaveraceae } \\
\hline Argemone subfusiformis Ownbey & cardosanto & 9 & 0.39 & $\mathrm{~F}, \mathrm{M}^{*}, \mathrm{~V}$ \\
\hline \multicolumn{5}{|l|}{ Passifloraceae } \\
\hline Passiflora caerulea L. & pasionaria & 4 & - & $\mathrm{M}^{*}, \mathrm{~N}^{*}$ \\
\hline \multicolumn{5}{|l|}{ Plantaginaceae } \\
\hline Plantago tomentosa Lam. & Ilantén & 12 & 0.37 & $F, M^{*}$ \\
\hline \multicolumn{5}{|l|}{ Poaceae } \\
\hline Avena sativa $\mathrm{L}$. & avena & 1 & & $\mathrm{~F}$ \\
\hline Cenchrus ciliaris $\mathrm{L}$. & \begin{tabular}{|l|} 
pasto, buffer \\
grass
\end{tabular} & 1 & & $\mathrm{~F}$ \\
\hline Panicum maximus Jacq. & pasto pani & 1 & & $\mathrm{~F}$ \\
\hline Sorghum sp. & *sorgo & 1 & & $\mathrm{~F}$ \\
\hline Zea mays L. & *maíz, choclo & 8 & & $F^{*}, V$ \\
\hline \multicolumn{5}{|l|}{ Polygonaceae } \\
\hline Rumex sp. & *lengua de vaca & 1 & - & $\mathrm{F}, \mathrm{M}^{*}$ \\
\hline Ruprechtia apetala Wedd. & $\begin{array}{l}\text { juda, manzano } \\
\text { del Campo }\end{array}$ & 42 & 1.13 & $\begin{array}{l}\mathrm{F}, \mathrm{M}^{*}, \mathrm{D} \\
\mathrm{V}, \mathrm{C}\end{array}$ \\
\hline \multicolumn{5}{|l|}{ Portulacaceae } \\
\hline Portulaca oleracea L. & verdolaga & 33 & 0.64 & $\mathrm{~F}, \mathrm{~N}^{*}$ \\
\hline \multicolumn{5}{|l|}{ Rhamnaceae } \\
\hline Condalia microphylla Cav. & piquillín & 37 & 1.01 & $\begin{array}{l}\mathrm{F}, M^{*}, D^{*}, \\
N^{*}\end{array}$ \\
\hline Ziziphus mistol Griseb. & mistol & 47 & 1.51 & \begin{tabular}{|l}
$\mathrm{F}, \mathrm{M}^{*}, \mathrm{D}$ \\
$\mathrm{V}, \mathrm{FI}^{*}, \mathrm{~N}^{*}$ \\
\end{tabular} \\
\hline \multicolumn{5}{|l|}{ Ranunculaceae } \\
\hline Clematis campestris A.St.-Hil. & barba de viejo & 6 & 0.1 & $\mathrm{~F}$ \\
\hline \multicolumn{5}{|l|}{ Rutaceae } \\
\hline Zanthoxylum coco Gillies ex Hook.f \& Arn. & coco & 2 & - & $D^{*}$ \\
\hline \multicolumn{5}{|l|}{ Salicaceae } \\
\hline Salix humboldtiana Willd. & sauce & 1 & - & $\mathrm{D}$ \\
\hline \multicolumn{5}{|l|}{ Santalaceae } \\
\hline Jodina rhombifolia (Hook. \& Arn.) Reissek & peje & 6 & 0.43 & $\begin{array}{l}\text { F, M* }{ }^{*}, \mathrm{D} \\
\text { V, Sh, Fl }\end{array}$ \\
\hline \multicolumn{5}{|l|}{ Sapindaceae } \\
\hline Cardiospermum halicacabum L. & globito, pedorra & 8 & 0.22 & $\mathrm{~F}, \mathrm{M}^{*}$ \\
\hline \multicolumn{5}{|l|}{ Simaroubaceae } \\
\hline Castela coccinea Griseb. & mistol del zorro & 17 & 0.25 & $\mathrm{~F}$ \\
\hline \multicolumn{5}{|l|}{ Solanaceae } \\
\hline Capsicum chacoense Hunz. & ají & 26 & 0.88 & $\mathrm{~F}, \mathrm{~N}^{*}$ \\
\hline Cestrum parqui (Lam.) L’Hér & duraznillo negro & 4 & 0.24 & $\begin{array}{l}\mathrm{F}, \mathrm{M}^{*}, \mathrm{D} \\
\mathrm{V}\end{array}$ \\
\hline Grabowskia boerhaaviifolia (L.f.) Schltdl. & fruto de paloma & 10 & 0.13 & $\mathrm{~F}$ \\
\hline
\end{tabular}


Trillo et al. - Perceptions and Use of Native Forests in the Arid Chaco of Córdoba, Argentina

\begin{tabular}{|c|c|c|c|c|}
\hline Lycium ciliatum Schltdl. & $\begin{array}{l}\text { piquillín de } \\
\text { la víbora }\end{array}$ & 8 & 0.12 & $\mathrm{~F}, \mathrm{M}^{*}, \mathrm{~V}$ \\
\hline Lycium elongatum Miers & gualeguay & 8 & 0.11 & $\mathrm{~F}$ \\
\hline Nicotiana glauca Graham & palán-palán & 8 & 0.53 & $F, M^{*}, V$ \\
\hline Nicotiana longiflora Cav. & flor de sapo & 1 & - & $\mathrm{M}^{*}$ \\
\hline Nierembergia linariifolia Graham & chuscho & 3 & 0.06 & $\mathrm{~F}$ \\
\hline Salpichroa origanifolia (Lam.) Baill. & uvita del campo & 1 & 0.03 & $\mathrm{~F}, \mathrm{~N}^{*}$ \\
\hline Solanum argentinum Bitter \& Lillo & $\begin{array}{l}\text { duraznillo } \\
\text { del burro }\end{array}$ & 15 & 0.29 & $\mathrm{~F}, \mathrm{~T}$ \\
\hline Solanum elaeagnifolium Cav. & quillo & 1 & 0.08 & $\mathrm{~F}, \mathrm{So}^{*}$ \\
\hline \multicolumn{5}{|l|}{ Urticaceae } \\
\hline Urtica urens L. & ortiga & 1 & - & $\mathrm{M}^{*}$ \\
\hline \multicolumn{5}{|l|}{ Verbenaceae } \\
\hline Aloysia gratissima (Gillies \& Hook.) Tronc. & palo amarillo & 30 & 0.97 & $F, D, M^{*}$ \\
\hline $\begin{array}{l}\text { Junellia hookeriana (Covas \& Schnack) N.O'Leary } \\
\text { \& P.Peralta }\end{array}$ & violeta & 3 & 0.08 & $\mathrm{~F}$ \\
\hline Lippia integrifolia (Griseb.) Hieron. & incayuyo & 41 & 1.34 & $\begin{array}{l}\mathrm{F}, \mathrm{M}^{*}, \mathrm{D}, \\
\mathrm{V}\end{array}$ \\
\hline Lippia turbinata Griseb. & poleo & 42 & 1.34 & $\begin{array}{l}\mathrm{F}, \mathrm{M}^{*}, \mathrm{D} \\
\mathrm{V}\end{array}$ \\
\hline \multicolumn{5}{|l|}{ Zygophyllaceae } \\
\hline Larrea cuneifolia Cav. & jarilla & 2 & 1.71 & $\mathrm{M}, \mathrm{D}^{*}, \mathrm{FI}$ \\
\hline Larrea divaricata Cav. & jarilla & 51 & 1.71 & $\begin{array}{l}\mathrm{F}, \mathrm{M}^{*}, \\
\mathrm{D}, \mathrm{V}, \\
\mathrm{S}, \mathrm{FI}, \mathrm{C}, \mathrm{T}, \\
\mathrm{Fu}^{*}\end{array}$ \\
\hline Porlieria microphylla (Baill.) Descole, O’Donell \& Lourteig & cucharero & 5 & 0.18 & $\mathrm{~F}, \mathrm{M}^{*}, \mathrm{~V}, \mathrm{FI}$ \\
\hline \multicolumn{5}{|l|}{ (Class Insecta) Dactylopidae } \\
\hline Dactylopius sp. & cochinilla & 1 & & $\mathrm{D}$ \\
\hline \multicolumn{5}{|l|}{ (Class Agaricomycetes) Ganodermataceae } \\
\hline Ganoderma lucidum (Curtis) P.Karst & polvo de San Juan & 1 & & $\mathrm{~V}$ \\
\hline \multicolumn{5}{|l|}{ (Class Sauropsida) Boeide } \\
\hline Boa constrictor occidentalis Philippi. 1873 & ampalagua & 2 & & V \\
\hline
\end{tabular}

cies showed a reduced number of citations (Table 1$)$. Most of the species of wide consensus $(n=15)$ were woody plants (tree or shrub) and presented at least five different uses (Table 1). Moreover, a high cultural value was confirmed for these 20 species by the herbarium.

The number of species mentioned for different uses was variable. Medicinal plants showed a higher number of citations (an average of 16 species per informant), while dyeing and nutritional plants were less cited (an average of two species).

Table 1 shows that the group of forage plant species is the most frequently mentioned. A total of 106 (100 wild and 4 cultivated) species from 47 botanical families were men- tioned, with an average number of cited species per informant of 22.1 ( $S D=16.5 ; C V=0.7$ ). Three forage plants (Prosopis spp., algarrobos; Ziziphus mistol Griseb., mistol; and S. lorentzii, orco quebracho) were most frequently mentioned as "excellent" forage, even by people with little or no experience with the forest who only recognized a few plants. In addition, these species presented a high cultural value (Table 1). Forage plants were classified in three groups by the residents: (1) those collected in the summer, like the fruits of Prosopis spp. and Z. mistol; (2) those for cutting or batting down the foliage (S. lorentzii, Ligaria cuneifolia (Ruiz \& Pav.) Tiegh., Schinus fasciculata (Griseb.) I.M.Johnst., R. apetala, and Tillandsia spp.); and (3) those that are bought or cultivated as Medicago sativa L., Zea mays L., Avena sativa L., Sorghum spp., and Pani- 

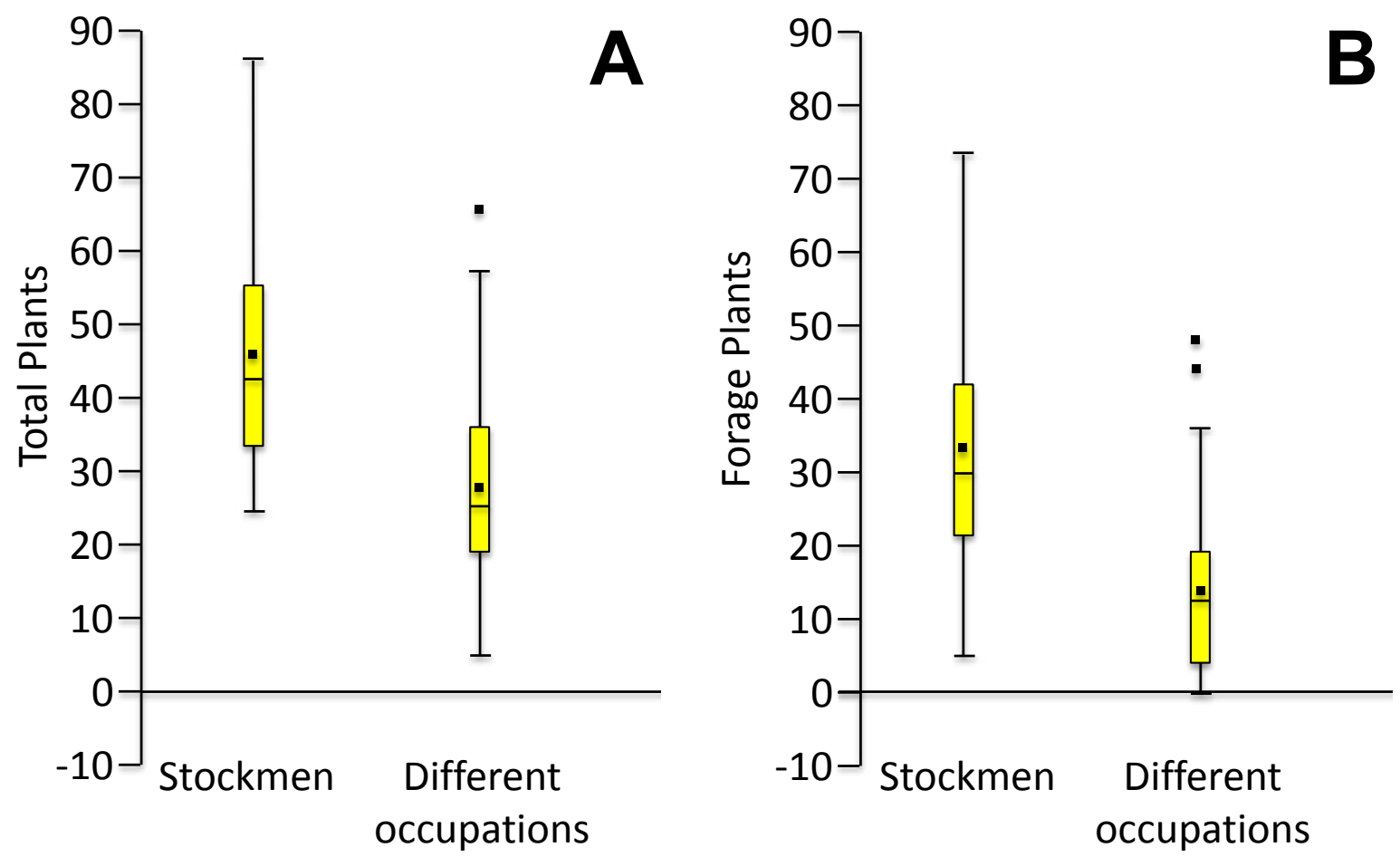

Figure 2. Box-plots for the data recorded on overall and forage plants known by resident stockmen and different occupation in the dry forest of the Chaco region, Córdoba, Argentina. $(\mathbf{A})$ Total number of known plants $(\mathrm{t}=4.77 ; \mathrm{p}=$ 0.0001). (B) Total number of known forage plants $(t=5.41 ; p=0.0001)$.

cum maximum Jacq. Plants in the last category are used in dry years or when forest fires make forage unavailable.

More than 100 species were cited by Río and Achával (1905), and this knowledge (e.g., plant names and uses) is currently available in the region (see Table 1).

\section{Practices associated with forage plants}

Stockmen carry out ancestral practices associated with the use of forage plants. Four out of the 37 informants mentioned that they regularly collect dry fruits under natural conditions from algarrobos and mistol, and that they place them in bags of $50 \mathrm{~kg}$. The bags are stored during the winter in structures near the house and the corrals. Only one resident mentioned the construction of a pirgua, a container manufactured as a basket using branches of L. divaricata to dry fruits. This structure $(1.3 \mathrm{~m}$ tall and 3 $\mathrm{m}$ circumference) can be used for about two years. Because cows and horses are particularly esteemed, they may receive food supplement of $M$. sativa or fruits of Prosopis spp.

Most stockmen (90\%) mentioned that they use corrals or cercos constructed with a support of Prosopis torquata (Lag.) DC. because this species produces the hardest wood. Then they add branches of $A$. caven, $A$. gilliesi, $A$. aroma, C. iguanaea, S. fasciculata, R. apetala, L. divaricata, and $A$. grattisima. The cercos usually enclose an area of 2 to 10 hectares that is not used during the rainy season (Spring and Summer) to allow forage to accumulate to be used during Winter. All the cercos have a hollow to collect rain water for animals. When the water disappears during the winter, the livestock are guided to the river or water from the family well is used.

Most of the informants (78.6\%) used three types of forage: collected, cut, and bought or cultivated. A total of $16.7 \%$ fed the livestock only with plants from the forest and never bought supplementary products, and only $4.8 \%$ exclusively bought forage. The stockmen used a wide spectrum of alternatives to feed the livestock, depending on the annual rainfall and their economic situation.

\section{Variation of knowledge of forage plants according to occupation}

The two types of residents, "stockmen" and "different occupation" (salaried people), differed significantly regarding their knowledge about forage plants. Figure 2 shows the average number of recognized plants by stockmen and salaried people, as well as the average number of forage plants known by these two groups. There are significant differences between groups $(t=4.77, p=0.0001$, and $t=5.41, p=0.0001$, respectively). The Chi-square of uniformity test showed there are no significant differences between the frequency of stockmen and inhabitants with other occupations $\left(X^{2}=1.57 ; p=0.21\right)$. 


\section{Trillo et al. - Perceptions and Use of Native Forests in the Arid Chaco of Córdoba, Argentina}

Table 2. Valuation of plants (according to a Likert-type scale) from dry Chaco forests of Córdoba, Argentina, comparing residents who are either stockmen or have different occupations. The values are the median for each category and for the different plant uses ( $3=$ higher valuation, $2=$ intermediate valuation, $1=$ low valuation).

\begin{tabular}{|l|c|c|l|}
\hline Plant uses & Stockmen (range) & Different occupation (range) & Statistics \\
\hline Forage & $3(1-3)$ & $3(1-3)$ & $\mathrm{W}=1155, \mathrm{p}=0.47$ \\
\hline Medical & $2(1-3)$ & $2(1-3)$ & $\mathrm{W}=1052, \mathrm{p}=0.46$ \\
\hline Dye & $2(1-3)$ & $1(1-3)$ & $\mathrm{W}=1036, \mathrm{p}=0.78$ \\
\hline Veterinarian & $1(1-3)$ & $1(1-3)$ & $\mathrm{W}=1081, \mathrm{p}=0.73$ \\
\hline Furniture & $3(1-3)$ & $3(1-3)$ & $\mathrm{W}=1079, \mathrm{p}=0.73$ \\
\hline Sensation & $3(2-3)$ & $2(2-3)$ & $\mathrm{W}=1163, \mathrm{p}=0.42$ \\
\hline
\end{tabular}

\section{Forest perception}

Table 2 shows the perception levels for forest plants categorized by different uses, comparing the two main occupations of the residents. Although there are some differences in the perception among plant uses, no statistical differences were found between residents categorized as stockmen or different occupation.

\section{Discussion}

Residents of the Guasapampa Valley possess an important ethnobotanical knowledge. This conclusion is based on the wide diversity of uses mentioned for 123 species, satisfying a great diversity of needs-some vital and others immaterial-but also on the comparison of this existing knowledge with the ethnobotanical knowledge registered over 100 years ago (Río \& Achával 1905, Trillo et al. 2010). Moreover, the knowledge of resources is of utmost importance because it both allows stockmen's daily activities and retains traditions and practices that identify them as criollos (Trillo 2010). According to Benz et al. (2000), the valuation of the forest as a venue for meeting residents' economic needs and the use of plants for other uses are factors that help a community maintain the practices inherited from past generations.

The main patterns highlighted in this study are two-fold. First, the knowledge of plants is not homogeneously distributed among rural residents of Guasapampa Valley but is rather related to the people's daily activities since stockmen showed a higher general knowledge of plant uses than inhabitants with different occupations, particularly on forage plants. Secondly, the inhabitants' positive perception of the forest values is generalized and independent of their main occupation.

These patterns can be compared from a global perspective and interpreted at the regional and local spatial scales. At a global scale, a similar direct correlation between residents' knowledge of plant resources and positive feelings toward forests was previously reported, independently of differences in their socioeconomic character- istics (e.g., Hayati et al. 2009, Karppinen 1998, Majumdar et al. 2008).

At the regional scale, the context is different because there are continuous expansions of agriculture over the few remaining forest fragments (Zak et al. 2008). Native vegetation (woody and herbaceous) is the principal source of forage plants for domestic livestock in the Chaco region (Río \& Achával 1905, Scarpa 2007). The practices observed in this study that are associated with the collection, cutting, and buying of forage are environmentally and culturally similar to activities of stockmen from other Chaco locations of Argentina (Arenas \& Scarpa 1999, Capparelli \& Raffino 1997, Morello \& Saravia Toledo 1959, Scarpa 2007). Traditional ecological knowledge, rural practices, and plant uses can be eroded in the short or middle time with consequences for rural people who obtain multiple services and resources that support their daily activities in a semiarid region.

At the local scale, inhabitants of Guasapampa Valley perceive the forest as a space to satisfy multiple daily requirements that not only involve economic aspects (mainly the maintenance of their livestock), but also satisfy medical and emotional necessities (e.g., passing of the crioIlos tradition to their children or living at a place that provides them with pleasant sensations). Stockmen have the knowledge and traditions to overcome dry years using different strategies for assuring the survival of their livestock. For example, goats consumed between $60-80 \%$ of native bushes during the dry season in a study of goat keepers performed a few kilometers away from Guasapampa Valley (Nai Bregaglio et al. 1999).Traditional knowledge may be useful when stockmen and inhabitants with different occupations share the landscape for different activities. Regarding the value of particular species in the Guasapampa Valley, it is interesting that the most valued ones are woody plants and few herbs. The higher comparative importance of woody plants for people is probably because they provide forage for animals during the whole year, but in particular during the dry season of this semiarid environment.

Forests worldwide can provide goods and many ecological services, but they are severely endangered, particu- 
larly those from Chaco (Zak et al. 2008). The modification of functional aspects of many plant species and their interactions in the Chaco region can be related to forest loss and fragmentation (Galetto et al. 2007, Grilli et al. 2012). Intensification of agriculture does not necessarily contribute to global hunger reduction. As Tscharntke et al. (2012) pointed out, food security and food sovereignty need to increase in areas where the hungry live, based on robust, eco-efficient approaches which incorporate natural biodiversity patterns and processes to increase sustainable productivity. The forest perception by the criollos and their plant knowledge evidenced in this study are linked to their practices, which relate to forest management decisions that are opposite to the process of agriculture expansion linked to the on-going forest loss in the Chaco region.

\section{Conclusion}

What are the most important implications of the observed relationships between plant knowledge, stockmen and salaried employees, and the generalized positive perception of the forest values in the Chaco-region? Results suggest that rural communities living in the Chaco forests preserve much of their knowledge and traditional practices associated with the collection, storage, and maintenance of many plant species, particularly regarding those that are used to feed animals. Secondly, ethnobotanical knowledge reflects links (products, needs, and emotions) between residents and the forest, mainly for stockmen because they depend on the forest to provide for livestock. Thirdly, despite significant cultural and economic changes during the last century that could have affected stockmen's traditions, they use the forests not only for daily activities but also to reinforce their identity as criollos.

Kleijn et al. (2009) proposed that agricultural intensification is the main driver of biodiversity decline, but at the same time, it helps sustain the growing world population. It is reasonable to recognize social conflicts due to changes in land use, particularly in the Chaco region. This study showed that it would be important to evaluate the residents' forest perception when defining conservation strategies on landscapes. In particular, residents consider the forests to be very significant places in the economic sense and also maintain a strong emotional link with them. These results might have relevant connotations in environment conservation. This approach may be complementary at the moment of designing conservation strategies that incorporate knowledge, use of the available resources, and socio-economic characteristics of the Chaco region. Biodiversity and cultural conservation can be advocated not only for pragmatic reasons but also for ethical reasons.

\section{Acknowledgments}

We thank the studied communities for their hospitality and collaboration, Julia Galetto for English professional editing, and Secretaría de Ciencia y Técnica (SECyT) of the Universidad Nacional de Córdoba and CONICET for financial support. SC and LG are researchers from CONICET (Consejo Nacional de Investigaciones Científicas y Técnicas).

\section{Literature Cited}

Arenas, P. 1999. Morrenia odorata (Asclepiadaceae), an edible plant of the Gran Chaco. Economic Botany 53(1):89-97. dx.doi.org/10.1007/BF02860798

Arenas, P. 2000. Farmacopea y curación de enfermedades entre algunas etnias del Gran Chaco. Pp. 87118 in Farmacobotánica y Farmacognosia en Argentina (1980-1998). Edited by A.G. Amat. Ediciones Científicas Argentinas, La Plata, Buenos Aires, Argentina.

Arenas, P. \& G.F. Scarpa. 1999. Ethnobotany of Stetsonia coryne (Cactaceae), the "cardón" of the Gran Chaco. Haseltonia 6:42-51.

Barboza, G.E., J.-J. Cantero, C. Núñez, A. Pacciaroni \& L.A. Espinar. 2009. Medicinal plants: A general review and a phytochemical and ethnopharmacological screening of the native Argentine flora. Kurtziana 34(1-2):7-365.

Benz, B.F., J. Cevallos E., F. Santana M., J. Rosales A. \& S. Graf M. 2000. Losing knowledge about plant use in the Sierra de Manantlan Biosphere Reserve, México. Economic Botany 54(2):183-191. dx.doi.org/10.1007/ BF02907821

Bergamin, G. 1992. Conceptualización sobre marginalidad, tipología de productores y desarrollo para caracterizar el Chaco Árido. Pp. 13-17 in Sistemas Agroforestales para Pequeños Productores de Zonas Áridas. Edited by R. Coirini \& U.O. Karlin. Facultad de Ciencias Agropecuarias, Universidad Nacional de Córdoba, and Proyecto Desarrollo Agroforestal Deutsche Gesellschaft für Technische Zusammenarbeit, Córdoba, Argentina.

Berkes, F. 1999. Context of traditional ecological knowledge. Pp. 3-16 in Sacred Ecology: Traditional ecological knowledge and resource management. Taylor \& Francis, Philadelphia, Pennsylvania, U.S.A.

Bernard, H.R. 1995. Research Methods in Anthropology: Qualitative and quantitative approaches. $2^{\text {nd }}$ edition. Altamira Press, Lanham, Maryland, U.S.A. 


\section{Trillo et al. - Perceptions and Use of Native Forests in the Arid Chaco of Córdoba, Argentina}

Cabido, M. \& M.J. Pacha. 2002. Vegetación y Flora de la Reserva Natural Chancaní. Publicaciones Técnicas serie C. Agencia Córdoba Ambiente, Córdoba, Argentina.

Cabrera, A. 1976. Regiones fitogeográficas Argentinas. Enciclopedia Argentina de Agricultura y Jardinería. Volume 2, Part 1. Edited by R. Lorenzo. Editorial ACME, Buenos Aires, Argentina.

Capparelli, A. \& R. Raffino. 1997. La etnobotánica de "El Shincal" (Catamarca) y su importancia para la arqueología I: Recursos combustibles y madereros. Parodiana 10(1-2):181-188.

Celton, D.E. 1993. La Población de la Provincia de Córdoba a Fines del Siglo XVIII. Academia Nacional de Historia, Buenos Aires, Argentina.

Cunningam, A.B. 2001. Etnobotánica Aplicada: Pueblos, usos de plantas silvestres y conservación. Manual de la serie Pueblos y Plantas, Volumen 4. Fondo Mundial para la Naturaleza (WWF), Editorial Nordan-Comunidad, Montevideo, Uruguay.

Di Tada, L.E. \& E.H. Bucher, eds. 1996. Biodiversidad de la Provincia de Córdoba. Volumen 1. Fauna. Universidad Nacional de Río Cuarto, Córdoba, Argentina.

Díaz, R.O. 2007. Utilización de Pastizales Naturales. Encuentro Grupo Editor, Córdoba, Argentina.

FAO. 2009. Situación de los Bosques del Mundo 2009. Perspectivas Regionales: América Latina y el Caribe. Food and Agriculture Organization of the United Nations, Rome, Italy.

Filipov, A. 1997. La farmacopea natural en los sistemas terapéuticos de los indígenas Pilagá. Parodiana 10(12):35-74.

Galetto, L., R. Aguilar, M. Musicante, J. Astegiano, A. Ferreras, M. Jausoro, C. Torres, L. Ashworth \& C. Eynard. 2007. Fragmentación de hábitat, riqueza de polinizadores, polinización y reproducción de plantas nativas en el Bosque Chaqueño de Córdoba, Argentina. Ecología Austral 17(1):67-80. http://ref.scielo.org/w3hpcv

Gonzalez, L.M. 1999. Estimación de los Niveles de Desarrollo Socio-Demográfico en la Provincia de Córdoba: Su relación con la participación impositiva a los municipios. Colección de Tesis de Maestría, Universidad Nacional de Córdoba, Córdoba, Argentina.

Grilli, G., C. Urcelay \& L. Galetto. 2012. Forest fragment size and nutrient availability: Complex responses of mycorrhizal fungi in native-exotic hosts. Plant Ecology 213(1):155-165. dx.doi.org/10.1007/s11258-011-9966-3
Hayati, D., B. Abadi, R. Movahedi \& M. Heidari. 2009. An empirical model of factors affecting farmers' participation in natural resources conservational programs in Iran. Journal of Food, Agriculture \& Environment 7(1):201-207.

Hieronymus, J. 1882. Plantae Diaphoricae Florae Argentinae: Ó revista sistemática de las plantas medicinales, alimenticias ó de alguna otra utilidad y de las venenosas, qùe son indígenas de la República Argentina ó que, originarias de otros países se cultivan ó se crian espontáneamente en ella. Guillermo Kraft, Buenos Aires, Argentina. Idoyaga Molina, A. 2001. Lo sagrado en las terapias de las medicinas tradicionales del Noa y Cuyo. Scripta Ethnologica 23:9-75. www.redalyc.org/resumen.oa?id=14802301

Karppinen, H. 1998. Values and objectives of non-industrial private forest owners in Finland. Silva Fennica 32(1):43-59.

Kleijn, D., F. Kohler, A. Báldi, P. Batary, E.D. Concepción, Y. Clough, M. Díaz, D. Gabriel, A. Holzschuh, E. Knop, A. Kovács, E.J.P. Marshall, T. Tscharntke \& J. Verhulst. 2009. On the relationship between farmland biodiversity and land-use intensity in Europe. Proceedings of the Royal Society of London, Biology 276(1658):903-909. dx.doi. org/10.1098/rspb.2008.1509

Likert, R. 1932. A technique for the measurement of attitudes. Archives of Psychology 22(140):5-55.

Medeiros, M.F.T. 2009. Etnobotánica Historica: Principios e procedimientos. Serie: Estudios y Debates. NUPEEA/ Sociedad Brasilera de Etnobiología e Etnoecología, Recife, Brasil.

Majumdar, I., L. Teeter \& B. Butler. 2008. Characterizing family forest owners: A cluster analysis approach. Forest Science 54(2):176-184.

Morello, J. \& C. Saravia Toledo. 1959. El bosque chaqueño I. Paisaje primitivo, paisaje natural y paisaje cultural en el Oriente de Salta. Revista Agronómica del Noroeste Argentino 3:5-81.

Muiño, W.A. 2010. Ethnobotanical study of the rural population of the west of the Pampa Plain (Argentina). Ethnobotany Research \& Application 8:219-231.

Nai Bregaglio, M., U. Karlin \& R. Coirini. 1999. Respuesta de especies arbustivas forrajeras a tratamientos de desmonte selectivo en el Chaco Árido, Argentina. Multiquina 8:101-109.

Padua, J. 1994. Técnicas de Investigación Aplicadas a las Ciencias Sociales. Sección de Obras de Sociología, Fondo de Cultura Económica, México. 
Phillips, O. \& A.H. Gentry. 1993. The useful plants of Tambopata, Perú: II Additional hypothesis in quantitative ethnobotany. Economic Botany 47(1):33-43. www.jstor.org/ stable/4255480

Phillips, O., A.H. Gentry, C. Reynel, P. Wilkin \& C. GalvezDurand. 1994. Quantitative ethnobotany and Amazonian conservation. Conservation Biology 8(1):225-248. dx.doi. org/10.1046/j.1523-1739.1994.08010225.x

Rio, M.E. \& L. Achával. 1905. Geografía de la Provincia de Córdoba. Volumen II. Gobierno de la Provincia de Córdoba, Argentina.

Scarpa, G.F. 2007. Etnobotánica de los Criollos del oeste de Formosa: Conocimiento tradicional, valoración y manejo de las plantas forrajeras. Kurtziana 33(1):153-174.

Trillo, C. 2010. Valoración del Bosque y Uso de las Plantas Silvestres por Parte de los Pobladores del Valle de Guasapampa, Noroeste de la Provincia de Córdoba. Tesis doctoral, Facultad de Ciencias Exactas Físicas y $\mathrm{Na}$ turales, Universidad Nacional de Córdoba, Córdoba, Argentina.

Trillo, C., P. Demaio, S. Colantonio \& L. Galetto. 2007. Conocimiento actual de plantas tintóreas por los pobla- dores del valle de Guasapampa, provincia de Córdoba. Kurtziana 33(1):65-71.

Trillo, C., B. Arias Toledo, L. Galetto \& S. Colantonio. 2010. Persistence of the use of medicinal plants in rural communities of the Western Arid Chaco [Córdoba, Argentina]. The Open Complementary Medicinal Journal 2:8089. $\underline{\text { dx.doi.org/10.2174/1876391X01002010080 }}$

Tscharntke, T., Y. Clough, T.C. Wanger, L. Jackson, I. Motzke, I. Perfecto, J. Vandermeer \& A. Whitbread. 2012. Global food security, biodiversity conservation and the future of agricultural intensification. Biological Conservation 151(1):53-59. dx.doi.org/10.1016/j.biocon.2012.01.068

Zak, M.R. \& M. Cabido. 2002. Spatial patterns of the Chaco vegetation of Central Argentina: Integration of remote sensing and phytosociology. Applied Vegetation Science 5(2):213-226. www.jstor.org/stable/1479069

Zak, M.R., M. Cabido, D. Cáceres \& S. Díaz. 2008. What drives accelerated land cover change in central Argentina? Synergistic consequences of climatic, socioeconomic, and technological factors. Environment Management 42(2):181-189. dx.doi.org/10.1007/s00267-008-9101-y 